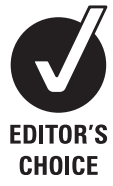

CHOICE

\title{
Ten problematical issues identified by pathology review for multidisciplinary gynaecological oncology meetings
}

\author{
W Glenn McCluggage
}

\section{Correspondence to}

Professor W Glenn McCluggage, Department of Pathology, Royal Group of Hospitals Trust, Grosvenor Road, Belfast, BT12 6BA, Northern Ireland, UK; glenn.mccluggage@ belfasttrust.hscni.net

Accepted 16 August 2011 Published Online First 19 October 2011

\begin{abstract}
Pathology review of gynaecological cancer specimens is often carried out as part of the working of gynaecological oncology multidisciplinary team meetings. This review describes the author's experience regarding the most common issues identified during this process. Ten subjects are covered; these range from histopathological interpretational errors to non-interpretational areas, for example, inappropriate use of the term 'microinvasive cervical carcinoma' and the use of inappropriate staging systems. This review is intended to be of practical use to the surgical pathologist reporting gynaecological cancer specimens.
\end{abstract}

In the UK and many other countries, multidisciplinary team meetings (MDTM), referred to as tumour board meetings in the USA, are a mandatory and established part of cancer management. ${ }^{1}$ In these forums, most cancers, with a few exceptions such as non-melanocytic skin malignancies, are discussed using a multidisciplinary approach with input from surgeons, oncologists, radiologists, pathologists, specialist nurses and a variety of other professionals involved in patient management.

As part of the MDTM process, review of pathology is often undertaken by specialist or subspecialist pathologists or general pathologists with a particular interest in an organ system. This may take several forms such as review by specialist pathologists of all cancers in a particular organ system in the same department or review of pathology from other departments, for example, review by specialist pathologists working in cancer centres of tumours reported at cancer units or diagnostic units.

Previous studies have identified more numerous and clinically significant diagnostic discrepancies in the field of gynaecological oncology than in other areas of pathology. ${ }^{2}$ Another study found that the most common diagnostic error in uterine tumour pathology was the overdiagnosis of early superficial myometrial invasion by endometrial carcinoma; ${ }^{3}$ this has largely been rendered irrelevant by the revised 2009 Fédération Internationale de Gynécologie et d'Obstétrique (FIGO) staging system for endometrial carcinomas, which combines intraendometrial carcinomas and carcinomas invading the inner half of the myometrium (IA and IB, respectively, in the 1988 FIGO staging system) into a single category of stage IA. ${ }^{4}$

In this review, I report my experience regarding the most common discrepancies identified by review of gynaecological cancer specimens in a cancer centre setting in the UK. This includes both review of internal cases and cases reported at neighbouring departments. All these cases are discussed at a weekly gynaecological oncology MDTM in Belfast, Northern Ireland. I discuss 10 problematical areas. Some of these represent histopathological interpretational errors while others are non-interpretational but may result in the incorrect information being relayed to the clinician. This review is intended to provide a practical aid to surgical pathologists reporting gynaecological cancer specimens.

\section{USE OF TERM 'MICROINVASIVE CERVICAL CARCINOMA'}

The measurement and staging of small cervical carcinomas is an issue that not uncommonly results in problems. Detailed criteria regarding the measurement of small cervical cancers, including multifocal neoplasms, has been provided in the Royal College of Pathologists dataset for histological reporting of cervical neoplasia ${ }^{5}$ in the UK and will not be repeated here.

In my experience, use of the term 'microinvasive cervical carcinoma' may result in confusion and I would recommend that the term 'microinvasive carcinoma' is not used but rather the tumour is measured and the appropriate FIGO stage provided on the pathology report in small cancers that have been removed by excisional biopsy, trachelectomy or hysterectomy. This applies to both squamous carcinomas and adenocarcinomas. I recommend avoidance of the term 'microinvasive carcinoma' because this terminology does not appear in the FIGO staging system for cervical cancer. Furthermore, use of the term 'microinvasive carcinoma' has different connotations in different institutions. In the UK, microinvasive carcinoma is considered to be synonymous with FIGO stage IA1 and IA2 disease in some institutions while in others the term is used only for FIGO stage IA1 tumours. In the USA, the term is largely synonymous with stage IA1 disease. The Society of Gynaecologic Oncology has its own definition of microinvasive carcinoma, which includes lesions up to a depth of $3 \mathrm{~mm}$ with no limit on the size of horizontal spread; neoplasms with lymphovascular invasion are excluded. ${ }^{6}$ In order to avoid confusion, the British Association of Gynaecological Pathologists working group has recommended in the Royal College of Pathologists dataset for histological reporting of cervical neoplasia a preference for avoiding the term 'microinvasive carcinoma' and for using the specific FIGO stage as a descriptor. ${ }^{5}$ 
Use of the term 'microinvasive carcinoma' may result in inappropriate patient management. For example, I have seen cases of FIGO stage IA2 carcinoma that have been reported by the pathologist as microinvasive carcinoma but the gynaecological oncologist has assumed this to represent FIGO stage IA1 disease and incorrect management has been instigated.

\section{WHICH STAGING SYSTEM TO USE FOR GYNAECOLOGICAL CANCERS}

Two staging systems are in widespread use for gynaecological cancers. These are the FIGO system, which is specific for gynaecological cancers and tumour-node-metastasis (TNM), which is applicable to all tumour sites. There is some controversy and confusion among specialists dealing with gynaecological cancers as to which staging system to use. Problems may arise as FIGO and TNM are not always directly comparable, particularly in the recording of lymph node involvement. In the FIGO system, lymph node involvement is incorporated into the final stage (except in cervical cancer), but in the TNM system this forms a separate staging component. For example, a FIGO stage IIIC endometrial or ovarian cancer (IIIC on the basis of lymph node involvement) may be pT1, lymph node involvement being recorded as a separate staging component, for example, $\mathrm{pN}$ 1. The British Association of Gynaecological Pathologists, British Gynaecological Cancer Society and the gynaecological clinical reference group of the National Cancer Intelligence Network recommend that FIGO staging is used for gynaecological cancers with recording of the lymph node status for cervical cancer as this is not included in the FIGO system. ${ }^{7}$ This may be done by providing a TNM stage (as well as a FIGO stage) for this cancer type only or by recording the lymph node status at the MDTM. These recommendations were made following a survey among participants in the national gynaecological pathology external quality assurance scheme and members of the British Gynaecological Cancer Society to determine which staging system for gynaecological cancers is most commonly used in the UK. The results of this survey showed that most gynaecological pathologists in the UK exclusively report gynaecological cancers using FIGO staging systems, and most gynaecological oncologists and other specialists dealing with patients with gynaecological malignancies likewise use FIGO. It is important that different centres use the same staging systems in order to facilitate comparison and exchange of data between centres and to allow for consistent transfer of data to cancer registries. Worldwide, most clinical trials and retrospective and prospective studies also use FIGO rather than TNM.

\section{ASSESSMENT OF CERVICAL INVOLVEMENT IN ENDOMETRIAL CARCINOMA}

There are several problematical issues regarding the histological assessment of cervical involvement in endometrial carcinoma, and a recent study revealed significant interobserver variation in the assessment of this among six specialist gynaecological pathologists. ${ }^{8}$

One problem is that the junction between the lower uterine segment/isthmus and the endocervix is not clearly defined. There are no histological landmarks that clearly delineate the junction and here there is an admixture of ciliated lower uterine segment endometrial glands and mucinous endocervical glands. In the interobserver variation study referred to, it was suggested that the uppermost mucinous gland might be taken as the junction between the cervix and the lower uterine segment, ${ }^{8}$ and I would recommend this until firm criteria are developed.
Endocervical glandular involvement by endometrial carcinoma may be subtle and difficult to recognise, especially if it involves the surface and forms a monolayer (figure 1). Because of this, there is a risk of the pathologist missing subtle endocervical glandular involvement. Problems may also arise in the distinction between endocervical surface involvement and so-called 'floaters' or artefactual tumour incorporation. ' With 'floaters' within the endocervical canal, it is usually relatively straightforward to ascertain that this does not represent true cervical involvement. However, problems arise when tumour is closely applied to the endocervical surface or embedded in granulation tissue secondary to implantation. Although cervical glandular involvement by endometrial carcinoma does not now denote stage II disease, this being defined by cervical stromal involvement in the 2009 FIGO staging system, ${ }^{4}$ many oncologists still administer vault brachytherapy when there is endocervical glandular involvement by endometrial carcinoma and so its recognition is still important.

There can also be significant issues in deciding whether tumour is confined to the cervical glandular epithelium or also involves the stroma. This is because normal endocervical epithelium as well as lining the surface invaginates to form crypts that lie within the superficial cervical stroma. Tumour may thus be present within the cervical stroma but still be confined to the glandular epithelium. It can be difficult in such cases to decide whether the neoplasm within the stroma is confined to pre-existing glandular elements, especially as endometrial adenocarcinomas invading the cervical stroma may not elicit a stromal reaction. Furthermore, some authors consider cervical glandular involvement to represent tumour confined to the surface only while others allow for underlying crypt involvement. I allow for underlying crypt involvement but the tumour should be clearly confined within the normal endocervical glandular field.

As discussed, there is a risk of missing focal microscopic endocervical surface involvement by endometrial carcinoma. We have previously described a common phenomenon involving the endocervical surface epithelium termed 'atypical reactive proliferation', which has the potential to be overdiagnosed as endocervical surface involvement by tumour, particularly when the changes are florid. ${ }^{10}$ This is a reactive phenomenon secondary to recent endometrial biopsy or curettage. ${ }^{10}$ The histological features, not all of which are present in every case, include

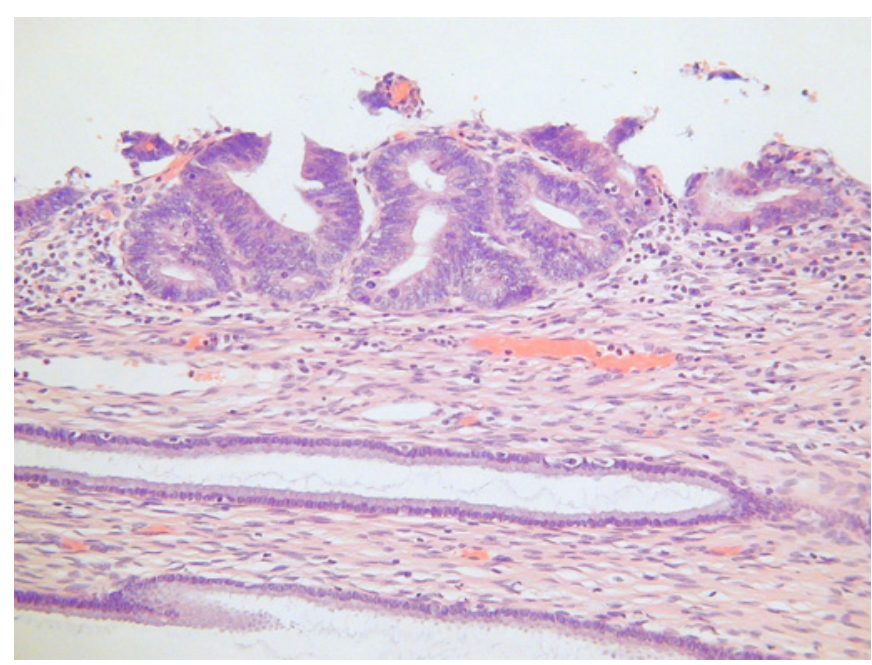

Figure 1 Endocervical glandular involvement by uterine endometrioid adenocarcinoma. 


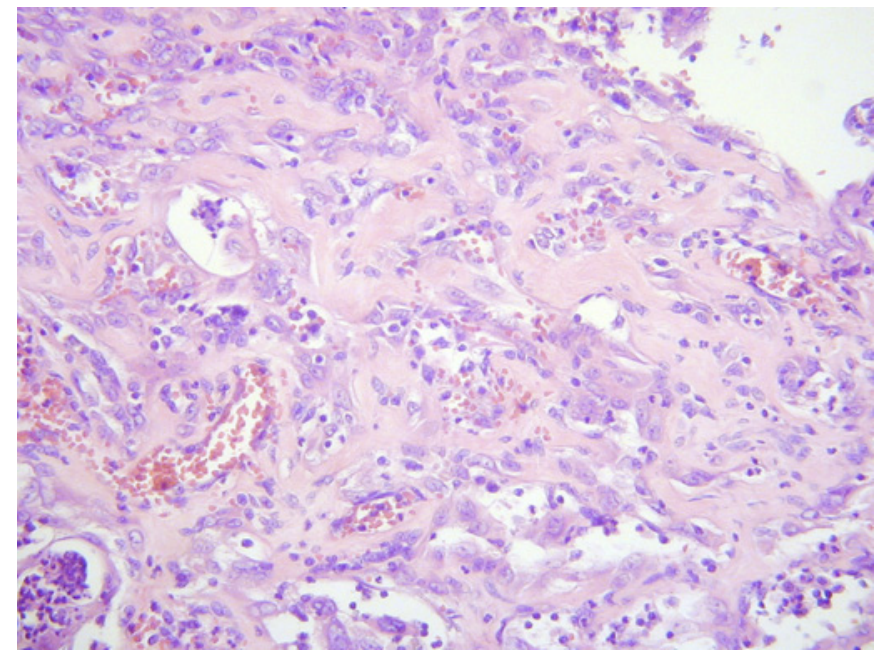

Figure 2 Florid reaction to previous endometrial biopsy with atypical epithelial cells embedded in fibrous stroma. The features may be misinterpreted as cervical involvement by endometrial adenocarcinoma.

nuclear stratification and multilayering with short micropapillary processes, squamoid change, hobnail cells and mild cytological atypia. Other features that may be present are surface erosion, clearing of the cytoplasm, fibrin deposition, an inflammatory cell infiltrate, fibrosis of the subepithelial tissue and extreme vascularity with a granulation tissue-like appearance. ${ }^{10}$ When benign glandular elements become entrapped within the fibrous stroma, the features may even mimic cervical stromal invasion by tumour. I have recently encountered some particularly florid examples that have resulted in considerable diagnostic difficulty (figure 2). Tubal or tuboendometrial metaplasia of the endocervical glands or superficial endometriosis in a patient with an endometrial carcinoma may also be mistaken for tumour involvement.

In most cases, cervical stromal involvement by endometrial carcinoma is easily recognised microscopically although, as already discussed, there may be problems in deciding whether tumour involves the endocervical stroma or the stroma of the lower uterine segment. However, a subtle 'burrowing' pattern of cervical stromal involvement can be misdiagnosed as cervical mesonephric remnants, tuboendometrial metaplasia or a coexistent premalignant or malignant endocervical glandular lesion. ${ }^{11}{ }^{12}$ With this burrowing pattern, the tumour infiltrates as 'naked' widely spaced, often cytologically bland, glands that lie beneath normal endocervical glands and do not elicit a stromal response (figure 3). At low power, the infiltrating glands, given their location and morphological features, can be misdiagnosed as mesonephric remnants, especially as they may have a somewhat linear arrangement and contain luminal eosinophilic 'colloid-like' material, both features of cervical mesonephric remnants. ${ }^{11} 12$ As well as mesonephric remnants, this pattern of infiltration may be misdiagnosed as a primary endocervical adenocarcinoma, cervical glandular intraepithelial neoplasia or tuboendometrial metaplasia. Occasional serous carcinomas of the uterine corpus also infiltrate the cervical stroma as widely spaced glands without eliciting a stromal response.

\section{CLASSIFICATION OF OVARIAN SEROUS CARCINOMA AS LOW GRADE OR HIGH GRADE}

It has now been convincingly demonstrated that there are two distinct types of ovarian serous carcinoma, low grade and high grade. ${ }^{13-17}$ Although termed 'low grade' and 'high grade' serous carcinoma, it is important to emphasise that these are not two grades of the same neoplasm but rather two distinct tumour types with different underlying pathogenesis, molecular events, behaviour and prognosis. High grade serous carcinoma is much more common than low grade. Low grade serous carcinoma is thought to arise in many cases in a stepwise fashion from a benign serous cystadenoma through a serous borderline tumour to an invasive low grade serous carcinoma. There is thus a well-defined adenoma-carcinoma sequence. In contrast, high grade serous carcinoma is not related to serous borderline tumour and was thought until recently to arise directly from the ovarian surface epithelium or the epithelium of cortical inclusion cysts with no well-defined precursor lesion. There is now emerging and quite compelling evidence that many high grade ovarian serous carcinomas actually originate from the epithelium of the distal fimbrial portion of the fallopian tube. ${ }^{18-23}$ Figure 4 illustrates the postulated pathways of development of low grade and high grade serous carcinoma.

Instead of grading ovarian serous carcinoma using a threetiered system (well, moderate, poor; grade 1, 2 or 3), pathologists should now classify these as high grade or low grade, and this practice has been recommended in the Royal College of Pathologists datasets for the histopathological reporting of neoplasms of the ovaries and fallopian tubes and primary carcinomas of the peritoneum in the $\mathrm{UK}^{24}$ however, some pathologists still persist in using old grading schemes.

The distinction between low and high grade serous carcinoma is based on morphology, the chief discriminator being the degree of nuclear atypia in the worst area of the tumour. ${ }^{25} 26$ The amount of mitotic activity is also taken into account. In low grade serous carcinoma, the nuclei are uniform with mild or at the most moderate atypia and less than or equal to 12 mitoses per 10 high power fields (the mitotic count is usually approximately two per 10 high power fields or less than this) (figure 5A). There is no necrosis or multinucleation. It is worth pointing out that prominent nucleoli and intracytoplasmic
Figure 3 Subtle pattern of cervical stromal invasion by endometrioid adenocarcinoma of uterine corpus with no stromal reaction (A). Eosinophilic luminal secretions are present and the features may be misinterpreted as representing mesonephric remnants, other benign cervical glandular lesions or a primary premalignant or malignant endocervical glandular lesion (B).
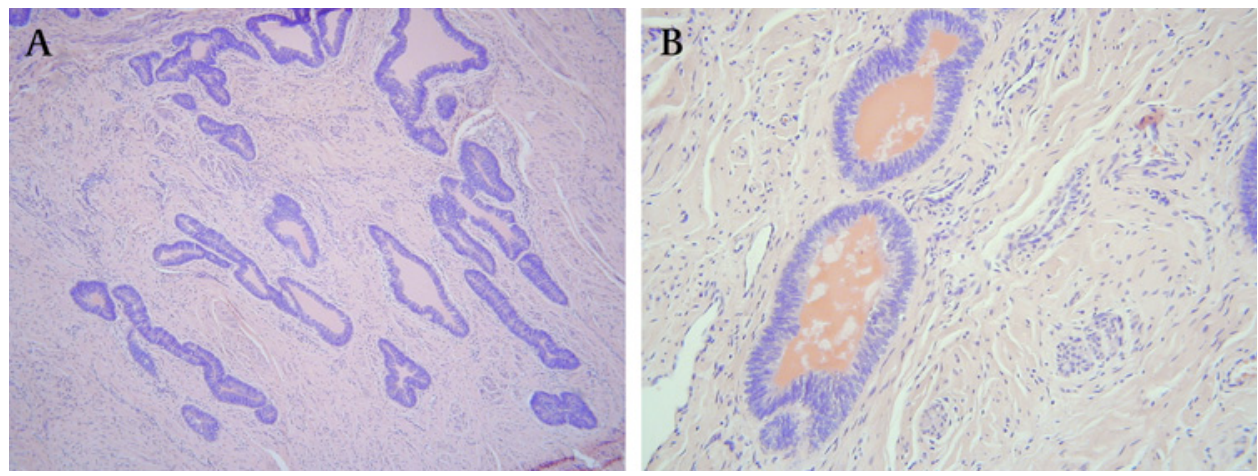
Figure 4 Developmental pathways of high grade and low grade ovarian serous carcinoma.

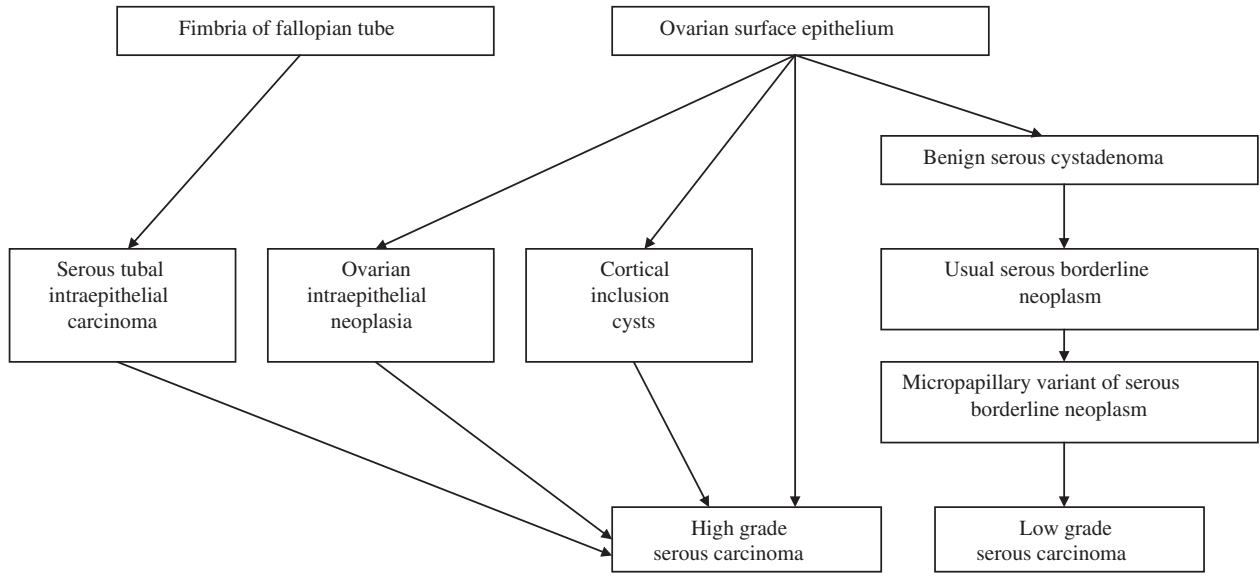

mucin are not uncommon in low grade serous carcinoma. ${ }^{14}$ High grade serous carcinoma exhibits moderate to marked nuclear atypia and greater than 12 mitoses per 10 high power fields (figure $5 \mathrm{~B}$ ). Necrosis and multinucleate cells are often present. It is generally not necessary to count mitotic figures as these are typically abundant in high grade serous carcinomas and difficult to find in low grade neoplasms. It is stressed that the distinction between low grade and high grade serous carcinoma is based on cytological and not architectural features (figure 5C). The classification of a serous carcinoma as low grade or high grade has been shown to be reproducible among pathologists. ${ }^{2526}$ Almost all serous carcinomas that would have previously been classified as moderately or poorly differentiated represent high grade neoplasms, while those that would have been classified as well differentiated may be either low grade or high grade using the two-tier classification. For example, some architecturally well-differentiated serous carcinomas have high nuclear grade and represent examples of high grade serous carcinoma.

The classification of a serous carcinoma as low grade or high grade has important clinical implications in that patients with high grade serous carcinoma are almost invariably treated by chemotherapy following surgical resection, even when stage I. In contrast, patients with stage I low grade serous carcinoma do not usually receive adjuvant therapy. In addition, there is now a tendency among medical oncologists not to treat advanced stage low grade serous carcinomas with adjuvant chemotherapy if total surgical debulking has been achieved. It is also important to distinguish between low grade and high grade serous carcinoma on a core biopsy because chemotherapy is considered to be relatively ineffective in low grade serous carcinoma and so oncologists are less likely to treat these neoplasms with upfront chemotherapy.

\section{DISTINCTION BETWEEN ATYPICAL HYPERPLASIA AND GRADE 1 ENDOMETRIOID ADENOCARCINOMA}

It may be difficult, or rarely even impossible, in a small biopsy specimen to distinguish between an atypical hyperplasia at the upper end of the spectrum and a grade 1 endometrioid adenocarcinoma. This is not surprising because these two lesions are part of a spectrum without clearly defined boundaries. In patients with a diagnosis of atypical hyperplasia on an endometrial biopsy, there is a significant risk of finding a low grade endometrioid adenocarcinoma in a subsequent hysterectomy specimen; this has varied between different studies but is usually in the order of $25-40 \% .{ }^{27-30}$ In my experience, in many cases this is not a result of progression or undersampling on biopsy but is a reflection of the fact that some pathologists are reluctant to make a diagnosis of carcinoma in an endometrial biopsy, particularly with a limited specimen or in a young patient. This is important because in many regions, patients with a diagnosis of atypical hyperplasia on an endometrial biopsy are not discussed at a MDTM.

There are several histological features that help to distinguish between atypical hyperplasia and grade 1 endometrioid adenocarcinoma. A desmoplastic stromal response is strong evidence of an adenocarcinoma, ${ }^{31}{ }^{32}$ but this is only seen in a minority of endometrial carcinomas in biopsy specimens. In my opinion, a back-to-back glandular architecture with complete exclusion of stroma is diagnostic of adenocarcinoma as in atypical hyperplasia stroma remains between glands ${ }^{33}$ (figure 6). Bridging between
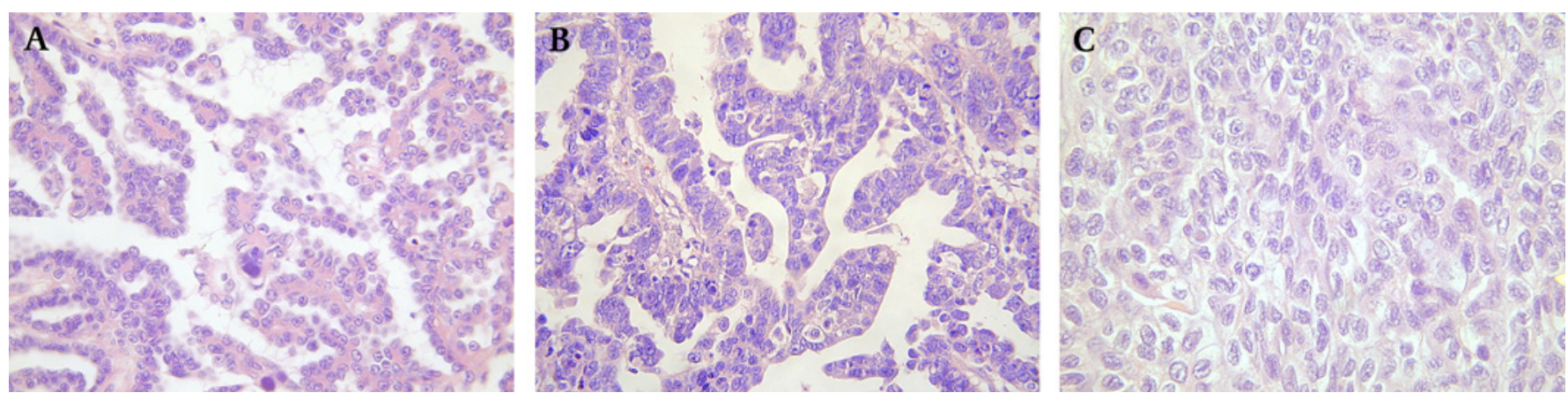

Figure 5 Low grade serous carcinoma composed of glands and papillae lined by bland cells with little in the way of nuclear atypia or mitotic activity (A). High grade serous carcinoma in which there is marked nuclear atypia and mitotic activity (B). Example of low grade serous carcinoma in which there is a solid architecture (C). 


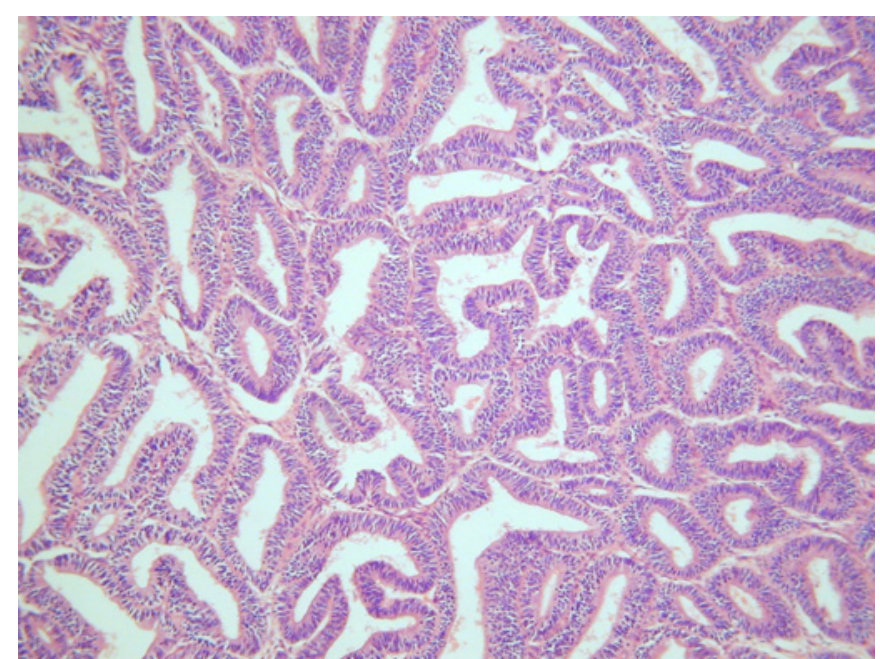

Figure 6 A back-to-back glandular architecture with stromal exclusion is diagnostic of adenocarcinoma in this endometrioid proliferation and excludes atypical hyperplasia.

adjacent glands resulting in a cribriform pattern and a papillary architecture are also in keeping with adenocarcinoma, as is the presence of luminal necrosis with polymorph infiltration.

\section{DISTINCTION BETWEEN ENDOMETRIAL AND CERVICAL ORIGIN FOR HIGH GRADE CARCINOMA}

It is not uncommon for there to be doubt as to whether an adenocarcinoma is of endometrial or cervical origin. There are a number of situations in which this occurs, such as when tumour is present in both an endometrial and a cervical biopsy or when the gynaecologist is not clear where the biopsy has been taken from. In such situations, radiology may assist but is often not helpful in determining the primary site. Establishing the site of origin is of considerable importance as cervical carcinomas are usually treated with radical hysterectomy or primary chemoradiation. Surgery, usually simple hysterectomy or sometimes modified radical hysterectomy when tumour involves the cervix, is the treatment of choice for endometrial carcinomas. Occasionally, even in the hysterectomy specimen it is difficult to ascertain whether a tumour has arisen in the endometrium or cervix and the decision whether or not to give adjuvant therapy and the nature of this may depend on the distinction.

A panel of immunohistochemical markers, comprising oestrogen receptor (ER), vimentin, carcinoembryonic antigen (CEA) and p16 may help with 'low grade' tumours when the differential lies between a low grade endometrioid adenocarcinoma and an endocervical adenocarcinoma of the usual type. ${ }^{34-36}$ Although there is some overlap, low grade endometrial adenocarcinomas of endometrioid type are usually diffusely positive with ER and vimentin and negative or focally positive with CEA and p16; there may be quite widespread p16 immunoreactivity but this is usually patchy with alternating positive and negative areas. ${ }^{37}$ In contrast, endocervical adenocarcinomas are usually diffusely positive with p16 and CEA and negative or focally positive with ER and vimentin; this panel of markers is well known to most pathologists.

A less well known, but significant, diagnostic dilemma is when a 'high grade' adenocarcinoma or an undifferentiated carcinoma presents in this way and the aforementioned panel of markers is of little value in such cases. Uterine serous carcinoma and undifferentiated carcinoma (discussed later) are aggressive endometrial carcinomas that may present in a cervical biopsy. If the panel of markers discussed is performed in an attempt to distinguish between an endometrial and a cervical origin, the pattern of diffuse p16 staining ( 16 is diffusely positive in most uterine serous carcinomas and some undifferentiated carcinomas) $)^{37-39}$ and negative or focal staining with ER in most uterine serous and undifferentiated carcinomas may result in misdiagnosis as a cervical primary with resultant inappropriate management. In this scenario, p53 staining and human papillomavirus (HPV) studies may be of value in that most uterine serous carcinomas and undifferentiated carcinomas exhibit aberrant p53 staining (diffusely positive or more uncommonly totally negative) ${ }^{40} 41$ and are HPV negative while most cervical carcinomas exhibit wild-type p53 staining (focal, weak and heterogenous pattern) and are HPV positive. P63 may also assist in that diffuse nuclear staining is suggestive of a poorly differentiated squamous carcinoma and a cervical origin, although squamous elements are present in some endometrial adenocarcinomas, especially of endometrioid type, and may be p63 positive

\section{MIXED CARCINOMAS OF THE OVARY}

According to WHO, a mixed carcinoma should only be diagnosed when the minor component makes up at least $10 \%$ of the neoplasm. ${ }^{42}$ However, I would recommend that all morphological subtypes within an ovarian carcinoma be documented and the percentages listed, even if the minor component accounts for less than $10 \%$. One point I wish to make is that true mixed carcinomas of the ovary (unlike in the uterus) are relatively uncommon, although they do occur, and there is a tendency to overdiagnose these. A combination of endometrioid and clear cell carcinoma occasionally occurs as both tumour types commonly arise in endometriosis. Neoplasms that are diagnosed as mixed serous and endometrioid or mixed serous and clear cell carcinoma mostly represent high grade serous carcinomas with areas that mimic endometrioid or clear cell carcinoma (figure 7); the combination of serous and endometrioid or serous and clear cell carcinoma is uncommon. WT1 may be useful in this regard as serous carcinomas of the ovary are usually diffusely positive while most endometrioid and clear cell carcinomas are negative. ${ }^{13} 1443-45$ The combinations of serous and undifferentiated or endometrioid and undifferentiated carcinoma sometimes occur. The former should be reported as a high grade serous

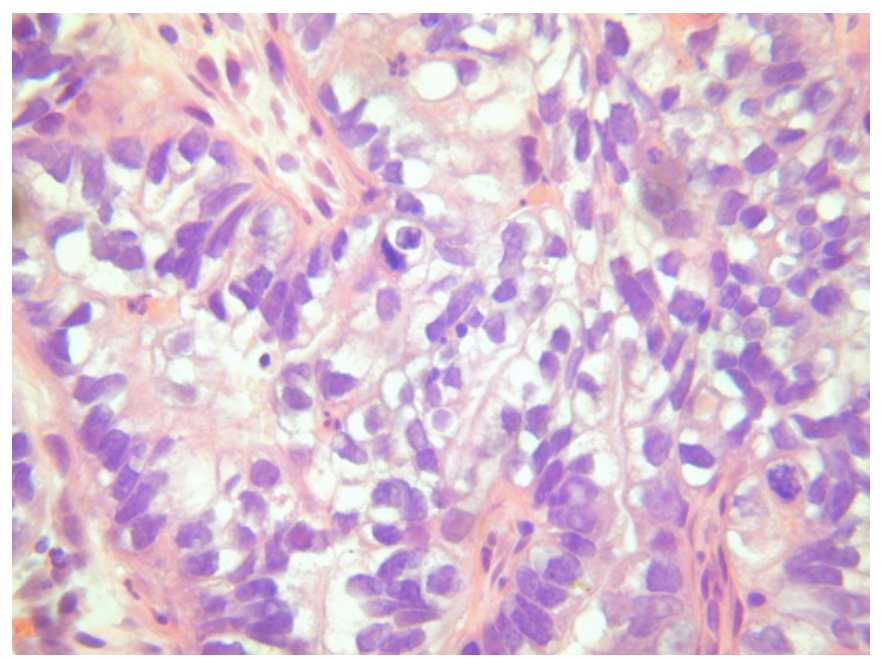

Figure 7 High grade ovarian serous carcinoma with clear cells; the clear cell areas may result in misdiagnosis as a clear cell carcinoma or mixed carcinoma with a clear cell component. 
carcinoma with a comment that undifferentiated areas are present and that these are in keeping with the extreme end of the spectrum of high grade serous carcinoma. As in the uterus, the combination of endometrioid and undifferentiated carcinoma occasionally occurs (discussed in detail later) and this probably represents dedifferentiation within a low grade endometrioid adenocarcinoma (dedifferentiated endometrioid adenocarcinoma).

\section{ASSESSMENT OF LYMPHOVASCULAR INVASION IN ENDOMETRIAL CARCINOMA}

In recent years, the assessment of lymphovascular invasion has become very important in endometrial adenocarcinoma. Lymphovascular invasion has been shown in many studies to be an independent prognostic factor in endometrial adenocarcinomas; ${ }^{46-51}$ blood vascular invasion seems to be more important than lymphovascular invasion. ${ }^{52}$ Of importance to the pathologist is that the presence or absence of lymphovascular invasion may be important in determining whether adjuvant therapy is administered following hysterectomy. For example, with a low grade endometrioid adenocarcinoma involving the outer half of the myometrium, vault brachytherapy is usually given while if there is associated lymphovascular invasion, external beam radiotherapy may also be given. In my experience, the assessment of lymphovasular invasion among pathologists is highly variable, with marked interobserver variability and there are a number of problematical issues.

Vascular involvement is typically most easily seen at the invasive front of the tumour (figure 8) and a perivascular lymphocytic infiltrate, including lymphoid aggregates, should raise the possibility of vessel involvement. Lymphovascular invasion should be distinguished from retraction artefact, which is not uncommonly seen in endometrial carcinomas. This distinction may be difficult but retraction artefact is often more widespread than true lymphovascular invasion and is characterised by a smooth round contour; with true vascular invasion the spaces typically have a more slit-like or angulated contour and are lined by endothelial cells. Markers such as CD31 (stains all vascular channels) and D2-40 (specifically stains lymphatic channels) may assist in identifying vascular invasion. ${ }^{52}$

Occasionally, small clusters of dyscohesive tumour cells with abundant eosinophilic cytoplasm that can mimic histiocytes are

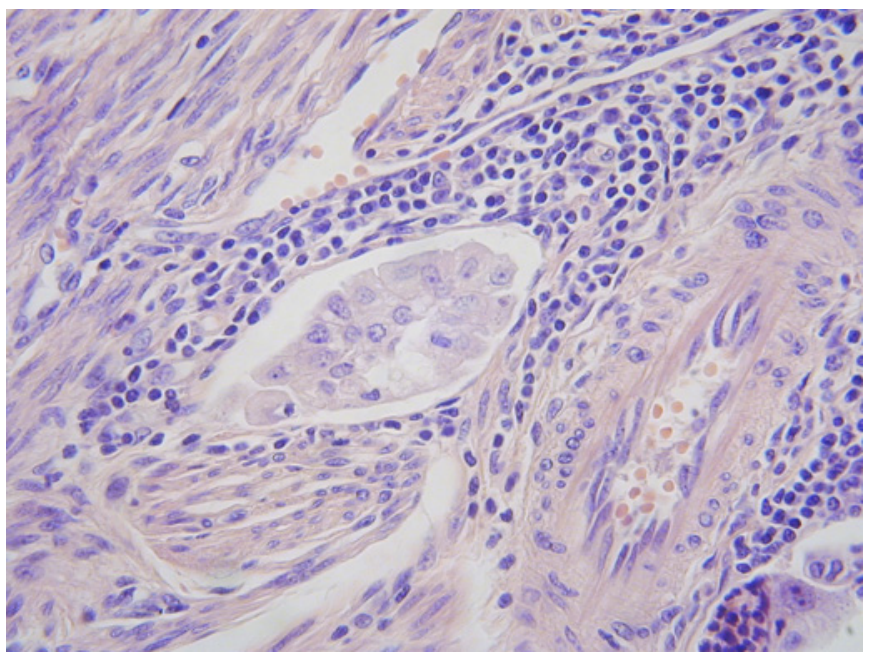

Figure 8 Lymphovascular invasion in endometrial adenocarcinoma. There is a surrounding lymphocytic infiltrate. present within vessels, and cytokeratins help in confirming their epithelial nature. ${ }^{51}$ True lymphovascular invasion should also be distinguished from artefactual vascular involvement, which is particularly common when there is marked tumour autolysis. Artefactual vascular invasion secondary to autolysis is characterised by 'smearing artefact' or the so-called toothpaste effect. The vascular invasion may be disproportionate in comparison with the stage and grade of the tumour and often the vessels involved are predominantly in the outer myometrium where tumour may also be seen smeared on the serosa.

The occurrence of artefactual vascular pseudoinvasion in total laparoscopic hysterectomy specimens using an intrauterine balloon manipulator has recently been highlighted. ${ }^{53-55}$ It has been suggested that this artefact, in which both benign and malignant endometrial tissue is displaced into vascular spaces, is the result of a closed positive pressure system created by the inflation of an intrauterine balloon following cautery occlusion of the fallopian tubes. ${ }^{53}$ Another suggestion is that the vascular pseudoinvasion is a grossing artefact secondary to mechanical disruption of friable polypoid tumour by the intrauterine balloon. ${ }^{54}$ Clues that this is an artefact include the contrast between the high volume of vascular invasion and the low tumour grade, the presence of stromal tissue accompanying the glands within vessels, preferential involvement of large thickwalled blood vessels in the outer myometrium and the absence of tumour adherence to the vessel wall (figure 9). Other features that may be seen in these specimens include disruption of the endometrial lining, endomyometrial clefts containing fragments of tumour, intratubal contaminants, nuclear crush artefact and intravascular inflammatory debris. ${ }^{55}$ Although these studies are limited by short follow-up and the significance of vascular pseudoinvasion is not clear, it is important to be aware of this artefact as misinterpretation can result in unnecessary adjuvant treatment. An increased incidence of positive peritoneal washings has also been demonstrated with total laparoscopic hysterectomy. ${ }^{55}$

A not uncommon pattern of myometrial infiltration by endometrioid adenocarcinoma has been referred to as microcystic, elongated, fragmented (MELF). ${ }^{56}$ In this pattern of invasion, there is a prominent fibromyxoid stromal reaction associated with the invasive glands (figure 10), which are also characterised by outpouchings from typical neoplastic glands that become

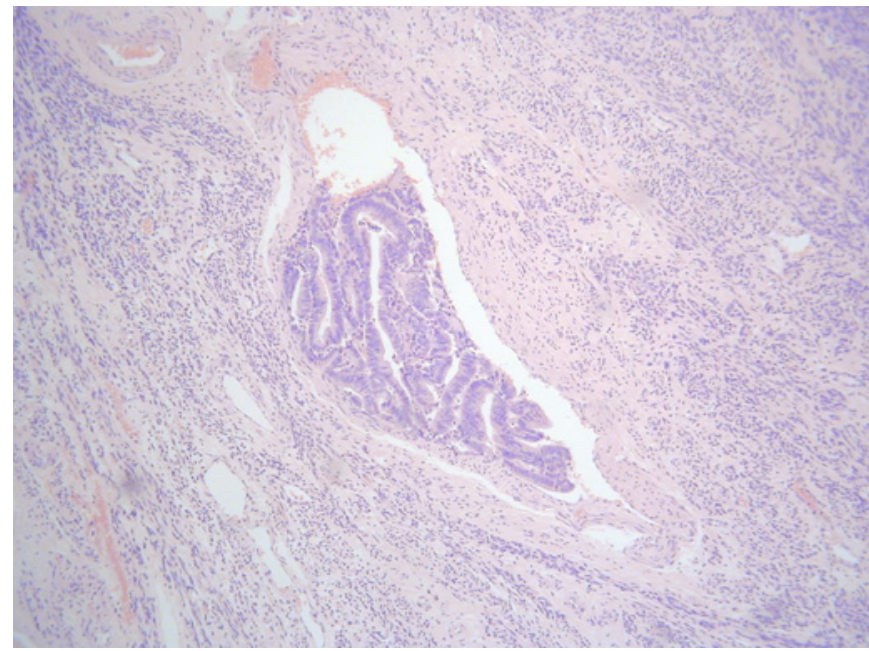

Figure 9 Artefactual involvement of vascular channels in endometrial adenocarcinoma secondary to the use of an intrauterine balloon manipulator. 
Figure 10 Microcystic elongated and fragmented (MELF) pattern of myometrial invasion in endometrioid adenocarcinoma (A). The glands lined by flattened cells may be mistaken for vascular channels with resultant overdiagnosis of vascular invasion (B).
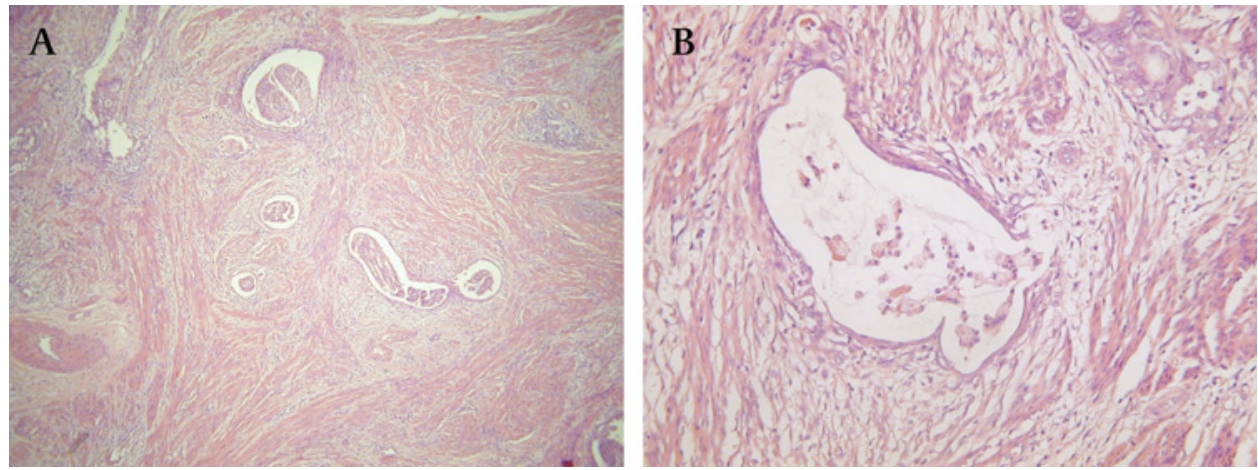

detached and lined by flattened epithelium, thus appearing as microcysts or slit-like spaces. The glands may become elongated or undergo fragmentation into small solid clusters or single cells that are surrounded by a fibromyxoid stromal reaction. These cells may lie deeper within the myometrium than the wellformed glands and can be overlooked, resulting in an underestimation of the depth of myometrial infiltration. This pattern of infiltration may also be mistaken for lymphovascular invasion because the glands that are lined by flattened epithelium can be mistaken for vascular channels (figure 10). Cytokeratin markers may be of value in distinguishing between glands lined by flattened cells and vascular spaces.

\section{MISDIAGNOSIS OF UNDIFFERENTIATED ENDOMETRIAL CARCINOMA AS GRADE 3 ENDOMETRIOID ADENOCARCINOMA OR SOME OTHER NEOPLASM}

The entity of undifferentiated endometrial carcinoma has recently gained increasing attention in the literature. ${ }^{57-60}$ In my experience, this is a not uncommon neoplasm (it has been found to account for approximately $9 \%$ of endometrial carcinomas) ${ }^{57}$ and it is included in the WHO classification of endometrial carcinomas. ${ }^{42}$ It has recently been defined as 'a tumour composed of medium or large cells with complete absence of squamous or glandular differentiation and with absent or minimal $(<10 \%)$ neuroendocrine differentiation'.57 This is an extremely aggressive variant of endometrial carcinoma, which may occur in pure form or in combination with and probably as a result of dedifferentiation in a low grade (grade 1 or 2 ) endometrioid adenocarcinoma (dedifferentiated endometrioid

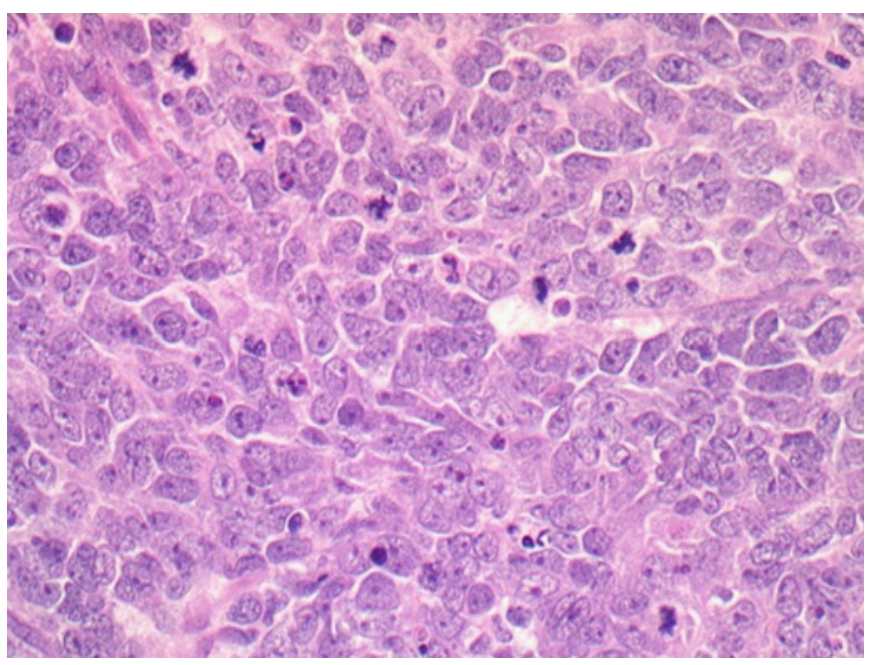

Figure 11 Undifferentiated endometrial carcinoma; misdiagnosis as grade 3 endometrioid adenocarcinoma is not uncommon. adenocarcinoma). Undifferentiated carcinoma is a specific histological entity and does not imply that the pathologist cannot come up with a more definitive diagnosis. The histological features are of a diffuse population of dyscohesive epithelioid cells with a characteristic monotonous and regular appearance (figure 11). The nuclei are often, but not always, large and vesicular. Mitoses are numerous. Other features found in a variable proportion of cases include a rhabdoid appearance, characterised by cells with eccentric nuclei and abundant eosinophilic cytoplasm, tumour giant cells, extensive necrosis and lymphovascular permeation and a focal myxoid stroma. The differential diagnosis of undifferentiated endometrial carcinoma includes grade 3 endometrioid adenocarcinoma, undifferentiated sarcoma, predominantly solid variants of serous carcinoma, carcinosarcoma, neuroendocrine carcinoma (a minor population of neuroendocrine cells of up to $10 \%$ is allowable in an undifferentiated endometrial carcinoma), lymphoma and other haematopoietic neoplasms, malignant melanoma and epithelioid sarcoma. Undifferentiated carcinomas are often only focally positive (characteristically $5-10 \%$ of cells) with cytokeratins, especially CK18, and epithelial membrane antigen, more consistently the latter. Even though staining is often focal, it is characteristically strong and this may be of use in diagnosis and in the distinction from other neoplasms.

In my experience, undifferentiated carcinomas are often misdiagnosed as a grade 3 endometrioid adenocarcinoma or even a grade 2 endometrioid adenocarcinoma if a component of low grade endometrioid adenocarcinoma is present. In grade 3

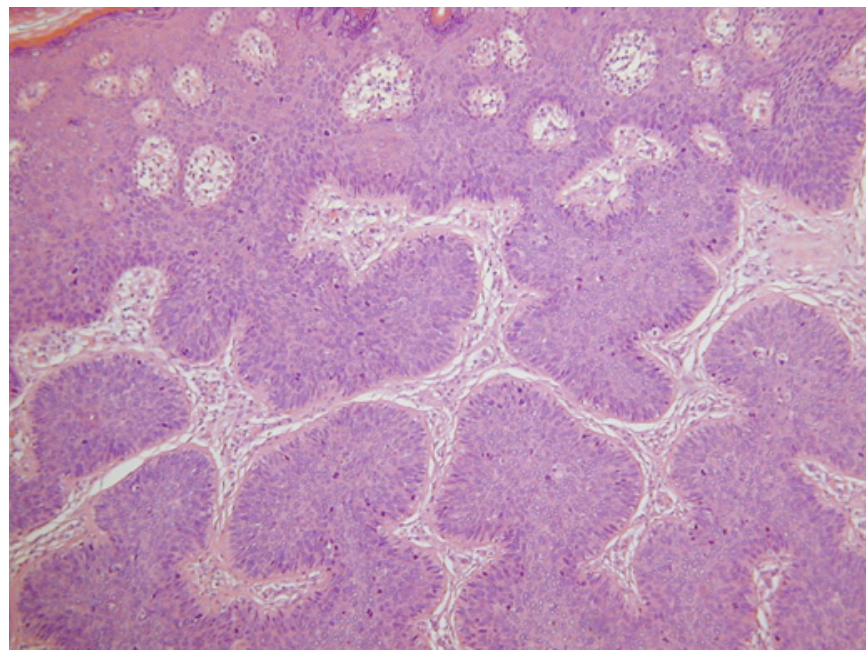

Figure 12 Classic vulval intraepithelial neoplasia with multiple downgrowths that are apparently detached from the surface squamous epithelium. This may result in overdiagnosis of invasive squamous carcinoma. 


\section{Take-home message}

Pathology review of gynaecological cancer specimens is often carried out as part of the working of gynaecological oncology multidisciplinary team meetings. Some errors are interpretational errors while others are non-interpretational but may result in the incorrect information being relayed to the clinician. Studies have identified more numerous and clinically significant diagnostic discrepancies in the field of gynaecological oncology than in other areas of pathology.

endometrioid adenocarcinoma, although much of the tumour is solid, there should be obvious glandular formation, which is absent in undifferentiated carcinoma (unless there is an associated component of low grade endometrioid adenocarcinoma); squamous elements may also be present in a grade 3 endometrioid adenocarcinoma. In dedifferentiated endometrioid adenocarcinoma, there is a component of grade 1 or 2 endometrioid adenocarcinoma as well as undifferentiated carcinoma; these should be reported as mixed endometrioid and undifferentiated carcinomas. Any component of undifferentiated carcinoma may be associated with aggressive behaviour. One study found the prognosis of undifferentiated endometrial carcinoma to be much worse than that of grade 3 endometrioid adenocarcinoma. ${ }^{57}$ In that study, undifferentiated endometrial carcinoma presented at an advanced stage in $54 \%$ of cases and $75 \%$ died of tumour; the corresponding figures for grade 3 endometrioid adenocarcinoma were $30 \%$ and $30 \%$. As well as being present in the primary tumour, undifferentiated elements may also be found in recurrent or metastatic endometrioid adenocarcinomas even when there is no undifferentiated component in the primary neoplasm within the uterine corpus. There is a suggestion that dedifferentiated endometrioid adenocarcinomas may be more prevalent in patients with Lynch syndrome (hereditary non-polyposis colorectal cancer syndrome). ${ }^{61}$

\section{OVERDIAGNOSIS OF INVASION IN CLASSIC VULVAL INTRAEPITHELIAL NEOPLASIA}

The most common type of vulval intraepithelial neoplasia (VIN), referred to as classic (undifferentiated, Bowenoid, HPV-related) type, may have an extremely 'hyperplastic' appearance with multiple downgrowths of the surface squamous epithelium into the subepithelial tissue. Due to tangentional sectioning, these may apparently become detached from the surface epithelium resulting in an impression of an invasive process (figure 12). The situation may be exacerbated by the involvement of skin appendage structures. While it is important not to miss an early invasive vulval squamous carcinoma (as lymphadenectomy is indicated with a depth of invasion of greater than $1 \mathrm{~mm}$ ), overdiagnosis of an invasive process may occur in such instances. This is an extremely problematical area and a recent study published in abstract form has shown significant interobserver variability in the diagnosis of early invasive vulval squamous carcinoma and in the measurement of the depth of invasion. ${ }^{62}$ The foci of apparent invasion are typically multiple and evenly spaced, have a bulbous appearance and a somewhat rounded contour with a regular edge and are composed of basaloid cells similar to those seen in the overlying VIN; they may be surrounded by basement membranelike material. In contrast, with true early invasion, the small foci of invasion typically have a more irregular outline and often a hypereosinophilic appearance with more abundant eosinophilic cytoplasm, in comparison with the basaloid appearance of the overlying VIN. A lymphocytic infiltrate is not particularly helpful as this may be seen surrounding downgrowths of VIN as well as around foci of early invasion. If a misdiagnosis of an invasive process is rendered, inguinal lymphadenectomy may be undertaken if the depth of invasion is measured as greater than $1 \mathrm{~mm}$.

\section{Competing interests None.}

Provenance and peer review Commissioned; externally peer reviewed.

\section{REFERENCES}

1. A policy framework for commissioning cancer services: a report by the expert advisory group on cancer to the chief medical officers of England and Wales. London; Department of Health, 1995.

2. Manion E, Cohen MB, Weydert J. Mandatory second opinion in surgical pathology referral material: clinical consequences of major disagreements. Am J Surg Pathol 2008;32:732-7.

3. Jacques SM, Qureshi F, Munkarah A, et al. Interinstitutional surgical pathology review in gynecologic oncology: II. Endometrial cancer in hysterectomy specimens. Int J Gynecol Pathol 1998;17:42-5.

4. Pecorelli S. Revised FIGO staging for carcinoma of the vulva, cervix and endometrium. Int J Gynaecol Obstet 2009;105:103-4.

5. Hirschowitz L, Ganesan R, Singh N, et al. Dataset for the histological reporting of cervical neoplasia. Royal College of Pathologists, April 2011.

6. Creasman WT. New gynecologic cancer staging. Gynecol Oncol 1995;58:198-202.

7. McCluggage WG, Hirschowitz L, Ganesan R, et al. Which staging system to use for gynaecological cancers: recommendations for practice in the United Kingdom. J Clin Pathol 2010:63:768-70.

8. McCluggage WG, Hirschowitz L, Wilson GE, et al. Significant variation in the assessment of cervical involvement in endometrial carcinoma: an interobserver variation study. Am J Surg Pathol 2011;35:289-94.

9. Jordan LB, Al-Nafussi A. Clinicopathological study of the pattern and significance of cervical involvement in cases of endometrial adenocarcinoma. Int J Gynecol Cancer 2002;12:42-8

10. Scott M, Lyness RW, McCluggage WG. Atypical reactive proliferation of endocervix: a common lesion associated with endometrial carcinoma and likely related to prior endometrial sampling. Mod Pathol 2006;19:470-4

11. Kalyanasundaram K, Ganesan R, Perunovic B, et al. Diffusely infiltrating endometrial carcinomas with no stromal response: report of a series, including cases with cervical and ovarian involvement and emphasis on the potential for misdiagnosis. Int J Surg Pathol 2010;18:138-43.

12. Tambouret R, Clement PB, Young RH. Endometrial endometrioid adenocarcinoma with a deceptive pattern of spread to the uterine cervix: a manifestation of stage $\mathrm{Ilb}$ endometrial carcinoma liable to be misinterpreted as an independent carcinoma or a benign lesion. Am J Surg Pathol 2003;27:1080-8.

13. McCluggage WG. My approach to and thoughts on the typing of ovarian carcinomas. J Clin Pathol 2008;61:152-63.

14. McCluggage WG. Morphological subtypes of ovarian carcinoma: a review with emphasis on new developments and pathogenesis. Pathology 2011;43:420-32.

15. Vang R, Shih leM, Kurman RJ. Ovarian low-grade and high-grade serous carcinoma: pathogenesis, clinicopathologic and molecular biologic features, and diagnostic problems. Adv Anat Pathol 2009;16:267-82.

16. McCluggage WG. The pathology of and controversial aspects of ovarian borderline tumours. Curr Opin Oncol 2010;22:462-72.

17. Seidman JD, Kurman RJ. Subclassification of serous borderline tumors of the ovary into benign and malignant types. A clinicopathologic study of 65 advanced stage cases. Am J Surg Pathol 1996;20:1331-45

18. Kindelberger DW, Lee $Y$, Miron A, et al. Intraepithelial carcinoma of the fimbria and pelvic serous carcinoma: evidence for a causal relationship. Am J Surg Pathol 2007:31:161-9.

19. Lee Y, Medeiros F, Mindelberger D, et al. Advances in the recognition of tubal intraepithelial carcinoma: applications to cancer screening and the pathogenesis of ovarian cancer. Adv Anat Pathol 2006;13:1-7.

20. Medeiros F, Muto MG, Lee $Y$, et al. The tubal fimbria is a preferred site for early adenocarcinoma in women with familial ovarian cancer syndrome. Am J Surg Pathol 2007:30:230-6.

21. Przybycin CG, Kurman RJ, Ronnett BM, et al. Are all pelvic (nonuterine) serous carcinomas of tubal origin? Am J Surg Pathol 2010;34:1407-16.

22. Lee $\mathbf{Y}$, Miron A, Drapkin R, et al. A candidate precursor to serous carcinoma that originates in the distal fallopian tube. J Pathol 2007;211:26-35.

23. Herrington CS, McCluggage WG. The emerging role of the distal fallopian tube and p53 in pelvic serous carcinogenesis. J Pathol 2010;220:5-6.

24. Wilkinson N, McCluggage WG. Datasets for the histopathological reporting of neoplasms of the ovaries and fallopian tubes and primary carcinomas of the peritoneum. Royal College of Pathologists, 2010.

25. Malpica A, Deavers MT, Lu K, et al. Grading ovarian serous carcinoma using a twotier system. Am J Surg Pathol 2004;28:496-504. 
26. Malpica A, Deavers MT, Tornos C, et al. Interobserver and intraobserver variability of a two-tier system for grading ovarian serous carcinoma. Am J Surg Pathol 2007:31:1168-74.

27. Leitao MM Jr, Han G, Lee LX, et al. Complex atypical hyperplasia of the uterus: characteristics and prediction of underlying carcinoma risk. Am J Obstet Gynecol 2010:203:349.e1-6.

28. Mittal K, Sebenik M, Irwin C, et al. Presence of endometrial adenocarcinoma in situ in complex atypical endometrial hyperplasia is associated with increased incidence of endometrial carcinoma in subsequent hysterectomy. Mod Pathol 2009:22:37-42.

29. Kurman R, Kaminski P, Norris $H$. The behaviour of endometrial hyperplasia: a long term study of "untreated" hyperplasia in 170 patients. Cancer 1985;56:403-12.

30. Trimble CL, Kauderer J, Zaino R, et al. Concurrent endometrial carcinoma in women with a biopsy diagnosis of atypical endometrial hyperplasia: a Gynecologic Oncology Group study. Cancer 2006;106:812-19.

31. Kurman RJ, Norris HJ. Evaluation of criteria for distinguishing atypical endometrial hyperplasia from well-differentiated carcinoma. Cancer 1982;15:2547-59.

32. Kurman RJ, Norris HJ. Endometrial stromal invasion in the diagnosis of welldifferentiated carcinoma. Am J Surg Pathol 1984;8:719-20.

33. McCluggage WG. My approach to the interpretation of endometrial biopsies and curettings. J Clin Pathol 2006;59:801-12.

34. McCluggage WG, Sumathi VP, McBride HA, et al. A panel of immunohistochemical stains, including carcinoembryonic antigen, vimentin and estrogen receptor aids the distinction between primary endometrial and endocervical adenocarcinomas. Int $J$ Gynecol Pathol 2002;21:11-15.

35. Castrillon DH, Lee KR, Nucci MR. Distinction between endometrial and endocervical adenocarcinoma: an immunohistochemical study. Int J Gynecol Pathol 2002;21:4-10

36. Kamoi S, Al Juboury ML, Akin MR, et al. Immunohistochemical staining in the distinction between endometrial and endocervical adenocarcinomas: another viewpoint. Int J Gynecol Pathol 2002;21:217-23.

37. Yemelyanova A, Ji H, Shih leM, et al. Utility of p16 expression for distinction of uterine serous carcinomas from endometrial endometrioid and endocervical adenocarcinomas: immunohistochemical analysis of 201 cases. Am J Surg Pathol 2009:33:1504-14.

38. Chiesa-Vottero AG, Malpica A, Deavers MT, et al. Immunohistochemical overexpression of p16 and p53 in uterine serous carcinoma and ovarian high-grade serous carcinoma. Int J Gynecol Pathol 2007;26:328-33.

39. Hirschowitz L, Ganesan R, McCluggage WG. WT1, p53 and hormone receptor expression in uterine serous carcinoma. Histopathology 2009;55:478-82.

40. McCluggage WG, Soslow RA, Gilks CB. Patterns of p53 immunoreactivity in endometrial carcinomas: "all or nothing" staining is of importance. Histopathology 2011;59:786-8.

41. Kobel M, Reuss A, Du Bois A, et al. The biological and clinical value of p53 expression in pelvic high-grade serous carcinomas. J Pathol 2010;222:191-8.

42. Tavassoli FA, Devilee P, eds. World Health Organisation Classification of Tumours Pathology and Genetics. Tumours of the Breast and Female Genital Organs. Lyon: IARC Press, 2003

43. Shimizu M, Toki T, Takagi Y, et al. Immunohistochemical detection of the Wilms tumor gene (WT1) in epithelial ovarian tumors. Int J Gynecol Pathol 2000:19:158-63.

44. Al-Hussaini M, Stockman A, Foster $\mathrm{H}$, et al. WT-1 assists in distinguishing ovarian from uterine serous carcinoma and in distinguishing between serous and endometrioid ovarian carcinoma. Histopathology 2004:44:109-15.
45. Goldstein NS, Uzieblo A. WT-1 immunoreactivity in uterine papillary serous carcinomas is different from ovarian serous carcinomas. Am J Clin Pathol 2002;117:541-5.

46. Stefansson IM, Salvesen HB, Immervoll H, et al. Prognostic impact of histological grade and vascular invasion compared with tumour cell proliferation in endometria carcinoma of endometrioid type. Histopathology 2004:44:472-9.

47. Gal D, Recio F0, Zamurovic D, et al. Lymphovascular space involvement a prognostic indicator in endometrial adenocarcinoma. Gynecol Oncol 1991;42:142-5.

48. Hanson MB, van Nagell JR Jr, Powell DE, et al. The prognostic significance of lymph-vascular space invasion in stage I endometrial cancer. Cancer 1985:55:1753-7.

49. Inoue $\mathbf{Y}$, Obata $\mathrm{K}$, Abe $\mathrm{K}$, et al. The prognostic significance of vascular invasion by endometrial carcinoma. Cancer 1996;78:1447-51.

50. Yamazawa K, Seki K, Matsui H, et al. Significance of perivascular lymphocytic infiltrates in endometrial carcinoma. Cancer 2001;91:1777-84

51. McKenney JK, Kong CS, Longacre TA. Endometrial adenocarcinoma associated with subtle lymph-vascular space invasion and lymph node metastasis: a histologic pattern mimicking intravascular and sinusoidal histiocytes. Int J Gynecol Pathol 2004;24:73-8.

52. Mannelqvist M, Stefansson I, Salvesen HB, et al. Importance of tumour cell invasion in blood and lymphatic vasculature among patients with endometrial carcinoma. Histopathology 2009:54:174-83.

53. Logani S, Herdman AV, Little JV, et al. Vascular "pseudo-invasion" in laparoscopic hysterectomy specimens: a diagnostic pitfall. Am J Surg Pathol 2008:32:560-5.

54. Kitahara S, Walsh C, Frumovitz M, et al. Vascular pseudoinvasion in laparoscopic hysterectomy specimens for endometrial carcinoma. A grossing artefact? Am J Surg Pathol 2009;33:298-303.

55. Krizova A, Clarke BA, Bernardini MQ, et al. Histologic artifacts in abdominal, vaginal laparoscopic, and robotic hysterectomy specimens: a blinded, retrospective review. Am J Surg Pathol 2011;35:115-26.

56. Murray SK, Young RH, Scully RE. Unusual epithelial and stromal changes in myoinvasive endometrioid adenocarcinoma: a study of their frequency, associated diagnostic problems, and prognostic significance. Int J Gynecol Pathol 2003:22:324-33

57. Silva EG, Deavers MT, Malpica A. Undifferentiated carcinoma of the endometrium: a review. Pathology 2007:39:134-8.

58. Altrabulsi B, Malpica A, Deavers MT, et al. Undifferentiated carcinoma of the endometrium. Am J Surg Pathol 2005;2:1316-21.

59. Tafe LJ, Garg K, Chew I, et al. Endometrial and ovarian carcinomas with undifferentiated components: clinically aggressive and frequently underrecognised neoplasms. Mod Pathol 2010;23:781-9.

60. Silva EG, Deavers MT, Bodurka DC, et al. Association of low grade endometrioid carcinoma of the uterus and ovary with undifferentiated carcinoma: a new type of dedifferentiated carcinoma? Int J Gynecol Pathol 2006;25:52-8.

61. Garg K, Leitao MM Jr, Kauff ND, et al. Selection of endometrial carcinomas for DNA mismatch repair protein immunohistochemistry using patient age and tumor morphology enhances detection of mismatch repair abnormalities. Am J Surg Pathol 2009;33:925-33

62. Abdel-Mesih A, Daya D, Onuma K, et al. Interobserver agreement among pathologists for assessing invasion in early vulvar squamous cell carcinoma: stil a diagnostic challenge. Mod Pathol 2010;23:232A. 